\title{
Correction: Evidence of inhibin/activin subunit betaC and betaE synthesis in normal human endometrial tissue
}

\author{
Ioannis Mylonas ${ }^{1,2^{*}}$, Ansgar Bruning ${ }^{1,2}$, Naim Shabani ${ }^{1,2}$, Susanne Kunze ${ }^{1,2}$, Markus S Kupka ${ }^{1,2}$
}

\section{Correction}

We have previously demonstrated the expression of inhibin subunits in endometrial tissues and used $\beta$-actin primer pairs to perform a PCR analysis loading control [1]. However, instead of using the mentioned $\beta$-actin primer pairs from a commercial molecular biology supplier, we used the $\beta$-actin primer pair listed in Table 1 . All depicted primer pairs in Table 1 were designed and established by our group, and synthesized by the supplier Biomers.net (Ulm, Germany). doi:10.1186/1477-7827-9-1

Cite this article as: Mylonas et al:: Correction: Evidence of inhibin/activin subunit betaC and betaE synthesis in normal human endometrial tissue. Reproductive Biology and Endocrinology 2011 9:1.

Table 1 Primer sequences and length of amplification products

\begin{tabular}{|c|c|c|c|}
\hline & Forward primer $\left(5^{\prime}-3^{\prime}\right)$ & Backward primer $\left(5^{\prime}-3^{\prime}\right)$ & Length \\
\hline Inhibin- $\alpha$ & CCGGCCATCCCAGCATACACGC & GAGTTGAGCGTCGGGCTCTC & $359 \mathrm{bp}$ \\
\hline Inhibin- $\beta A$ & TGCCCTTGCTTTGGCTGAGA & ACTITGCCCACATGAAGCTIT & $282 \mathrm{bp}$ \\
\hline Inhibin- $\beta B$ & GGCGAGCGGCGACTCAACCTAGA & CGTGTGGAAGGAGGAGGCAGAGC & $333 \mathrm{bp}$ \\
\hline Inhibin- $\beta C$ & GCAGCCCGGGTGAGAGTTGG & ACTGCACCCACAGGCCTC & $393 \mathrm{bp}$ \\
\hline Inhibin- $\beta E$ & AGCCCTTCCTAGAGCTTAAG & GCTGCAGCCACAGGCC & $404 \mathrm{bp}$ \\
\hline$\beta$-actin & GGAGAAGCTGTGCTACGTCG & CGCTCAGGAGGAGCAATGAT & $366 \mathrm{bp}$ \\
\hline
\end{tabular}

$\mathrm{bp}=$ base pairs

\section{Author details \\ ${ }^{1}$ Ludwig-Maximilians-University Munich, 1st Department of Obstetrics and Gynecology, Maistrasse 11, 80337 Munich, Germany. ${ }^{2}$ Department of Obstetrics and Gynecology, Klinikum Neuperlach, Munich, Germany.}

Received: 22 December 2010 Accepted: 5 January 2011

Published: 5 January 2011

\section{Reference}

1. Mylonas I, Brüning A, Shabani N, Kunze S, Kupka MS: Evidence of inhibin/ activin subunit betaC and betaE synthesis in normal human endometrial tissue. Reprod Biol Endocrinol 2010, 19(8):143.

\footnotetext{
* Correspondence: ioannis.mylonas@med.uni-muenchen.de ${ }^{1}$ Ludwig-Maximilians-University Munich, 1st Department of Obstetrics and Gynecology, Maistrasse 11, 80337 Munich, Germany

Full list of author information is available at the end of the article
}

Submit your next manuscript to BioMed Central and take full advantage of:

- Convenient online submission

- Thorough peer review

- No space constraints or color figure charges

- Immediate publication on acceptance

- Inclusion in PubMed, CAS, Scopus and Google Scholar

- Research which is freely available for redistribution

Submit your manuscript at www.biomedcentral.com/submit

C Biomed Central 